\title{
EVOLUÇÃO DA MORTALIDADE POR CÂNCER DE FÍGADO E COLORRETAL SEGUNDO SEXO E REGIÕES DO BRASIL DE 1990 A 2014.
}

\section{EVOLUTION OF MORTALITY DUE TO LIVER AND COLORECTAL CANCER ACCORDING TO SEX AND REGIONS OF BRAZIL, 1990 TO 2014.}

\author{
Mariana Castro Barros ${ }^{1}$, Ingrid Thaís Reis Pinheiro ${ }^{2}$, Leila Alves Oliveira ${ }^{3}$, \\ Wyllyane Rayana Chaves de Carvalho Santos ${ }^{4}$, Carolina Abreu de Carvalho ${ }^{5}$
}

RESUMO: O presente trabalho visa analisar a evolução da mortalidade por câncer de fígado e colorretal de acordo com o sexo e as regiões do Brasil, durante o período de 1990 a 2014 . Realizouse uma análise de séries temporais dos óbitos por câncer de fígado e vias biliares e colorretal, ocorridos no Brasil de 1990 a 2014. Os dados foram obtidos no Atlas On-line sobre Mortalidade do Instituto Nacional de Câncer (INCA). As taxas de mortalidade padronizadas (TMP) para a população brasileira e ajustadas por idade foram obtidas em cinco quadriênios: 1990-1994, 19951999, 2000-2004, 2005-2009, 2010-2014. Para verificar a tendência da mortalidade, empregou-se regressão linear simples. A magnitude de crescimento das TMP foram calculadas dividindo-se a taxa no último quinquênio pela taxa no primeiro. Observou-se tendência de aumento da TMP para os dois tipos de câncer analisados em ambos os sexos, com um crescimento mais expressivo no último quinquênio. Ao longo de toda a série histórica, a TMP por câncer de fígado e colorretal foi maior entre homens. A região Sul obteve maiores TMP para os dois tipos de câncer. Entretanto, a magnitude de crescimento da TMP por câncer de fígado foi maior na região Norte e por câncer colorretal na região Nordeste. As TMP por todos os tipos de câncer analisados apresentaram tendência de aumento no Brasil, com maior magnitude no último quinquênio. Destaca-se que, embora as TMP tenham sido maiores na região Sul, o crescimento ao longo do tempo foi maior em

\footnotetext{
${ }^{1}$ Discente do $7^{\circ}$ período do curso de Nutrição da Universidade CEUMA. Integrante do grupo de pesquisa "Núcleo de Estudo em Alimentação e Nutrição" da Universidade CEUMA. E-mail: cbarros.mariana@ gmail.com

${ }^{2}$ Discente do $5^{\circ}$ período do curso de Nutrição da Universidade CEUMA. Integrante do grupo de pesquisa "Núcleo de Estudo em Alimentação e Nutrição" da Universidade CEUMA. E-mail: ingridt977@ gmail.com

${ }^{3}$ Discente do $7^{\circ}$ período do curso de Nutrição da Universidade CEUMA. Integrante do grupo de pesquisa "Núcleo de Estudo em Alimentação e Nutrição" da Universidade CEUMA. E-mail: oliveira_alvesleila@ hotmail.com

${ }^{4}$ Docente do curso de Nutrição da Universidade CEUMA. Mestre em Saúde Coletiva pela Universidade Federal do Maranhão. Doutoranda em Saúde Coletiva na Universidade Federal do Maranhão. Pesquisadora do grupo de pesquisa "Núcleo de Estudo em Alimentação e Nutrição" da Universidade CEUMA. E-mail: wyllyane_rayana@ hotmail.com

${ }^{5}$ Docente do curso de Nutrição da Universidade CEUMA. Mestre em Ciência da Nutrição pela Universidade Federal de Viçosa. Doutoranda em Saúde Coletiva na Universidade Federal do Maranhão. Pesquisadora do grupo de pesquisa "Núcleo de Estudo em Alimentação e Nutrição" da Universidade CEUMA. E-mail: carolcarvalho91 @ gmail.com
} 
regiões menos desenvolvidas como o Norte e Nordeste, evidenciando a ocorrência de padrões regionais na distribuição e magnitude de crescimento das taxas.

PALAVRAS-CHAVE: Câncer de fígado. Câncer colorretal. Mortalidade.

ABSTRACT: This paper aims to analyze the evolution of mortality due to liver and colorectal cancer according to the sex and regions of Brazil during the period from 1990 to 2014. An analysis of time series of deaths from liver and bile duct cancer and colorectal cancer occurred in Brazil from 1990 to 2014. Data were obtained from the Mortality Atlas of the National Cancer Institute (INCA). Standardized mortality rates (SMR) for the Brazilian population and adjusted for age were obtained in five five-year periods: 1990-1994, 1995-1999, 2000-2004, 2005-2009, 2010-2014. To verify the trend of mortality, simple linear regression was used. The magnitude of TMP growth was calculated by dividing the rate in the last five years by the rate in the first five-years. There was an upward trend in PMT for the two types of cancer analyzed in both sexes, with a more significant growth in the last five years. Throughout the historical series TMP for liver and colorectal cancer was higher among men. The Southern region obtained higher TMP for both types of cancer. However, the magnitude of TMP growth due to liver cancer was higher in the North region and colorectal cancer in the Northeast region. The TMPs for all types of cancer analyzed showed a tendency to increase in Brazil, with greater magnitude in the last five years. It should be noted that, although PMT were higher in the South region, growth over time was higher in less developed regions such as the North and Northeast, evidencing the occurrence of regional patterns in the distribution and magnitude of growth rates.

KEYWORDS: Liver cancer. Colorectal cancer. Mortality.

\section{INTRODUÇÃO}

Estudos sobre a tendência da mortalidade por câncer no mundo têm mostrado que os resultados diferem de acordo com o nível de desenvolvimento dos países. Entre os países de alta renda, a tendência tem sido declinante, enquanto que, em países de média e baixa renda, como o Brasil, a taxa de mortalidade por câncer tem crescido (Vineis e Wild, 2014; Torre et al., 2016).

Estimativas globais sobre a mortalidade por câncer apontam que o câncer de fígado e colorretal estão entre os seis tipos de câncer que mais matam no mundo, tanto em países desenvolvidos, quanto em desenvolvimento (Ferlay et al., 2015).

As taxas de mortalidade por câncer de fígado e vias biliares e colorretal estão entre as 
maiores do Brasil. Estudos têm apontado o aumento da mortalidade por câncer de fígado e vias biliares no Brasil até 2010 (Amorim e Merchan-Hamann, 2013; Guimarães et al., 2015). A mortalidade por câncer de cólon e reto no Brasil no período de 1980 a 2009 também apresentou tendência de aumento linear (GuimarãEs et al., 2012). Todavia, tendências mais recentes (2010 a 2014) sobre a evolução desses tipos de câncer no país ainda não foram estudadas.

Em um país como dimensões continentais como o Brasil e com grandes discrepâncias regionais e sociais, é de se esperar que hajam diferenças na evolução da mortalidade por câncer. Girianelli et al. (2014) referiram os grandes contrastes na mortalidade por câncer de colo uterino e mama no Brasil devido à oferta e ao acesso ao rastreamento, diagnóstico e tratamento que se distribuem de forma desigual (Girianelli et al., 2014). Isso aponta para a relevância de se mapear as diferenças regionais quanto a outros tipos de câncer de alta mortalidade no Brasil.

O acompanhamento sobre a taxa de mortalidade por câncer permite monitorar e estabelecer um perfil sobre os tipos de câncer que mais matam. Dessa forma, é possível inferir a respeito dos fatores associados e elaborar estratégias de prevenção para redução da mortalidade por essas neoplasias.

Devido á alta mortalidade por câncer de fígado e vias biliares, cólon e reto no país e a necessidade de se manter atualizado o acompanhamento da evolução das taxas de mortalidade, o objetivo do presente estudo é analisar a evolução da mortalidade por câncer de fígado e vias biliares e colorretal no Brasil, durante o período de 1990 a 2014.

\section{MATERIAIS E MÉTODOS}

Realizou-se uma análise de séries temporais, tendo como unidade de observação os óbitos por câncer de fígado e vias biliares intra-hepáticas e o agrupamento fixo cólon e reto, ocorridos no Brasil no período de 1990 a 2014.

Os dados foram coletados no Atlas On-line sobre Mortalidade do Instituto Nacional de Câncer (INCA) ${ }^{6}$. Por meio deste site foi possível obter a taxa de mortalidade para cada tipo de câncer em estudo, ajustada por idade e pela população brasileira (a partir de dados dos censos do Instituto Brasileiro de Geografia e Estatística), por 100.000 habitantes.

As taxas de mortalidade foram obtidas em cinco quadriênios, a saber: 1990-1994, 1995 1999, 2000-2004, 2005-2009, 2010-2014. Para obtenção dos dados, foram selecionados como localização primária o fígado e vias biliares intra-hepáticas (C22) e o agrupamento fixo cólon e reto

\footnotetext{
${ }^{6}$ Disponível em:

https://mortalidade.inca.gov.br/MortalidadeWeb/pages/Modelo02/consultar.xhtml;jsessionid=6F80E015ED10602E7AE 2B8213BB000E8\#panelResultado.
} 
(C18-C20). Os quinquênios que incluíram o período de 1990 a 1999 foram ajustados para a população brasileira de 1991. O período de 2000 a 2009 foi ajustado para a população brasileira de 2000 e o último quinquênio (2010 a 2014) foi ajustado para a população brasileira de 2010.

Os dados foram digitados em uma planilha do Microsoft Excel® e, posteriormente, analisados no programa Stata ${ }^{\circledR}$ versão 14.0. Para verificar a tendência da mortalidade para os tipos de câncer estudados empregou-se regressão linear simples, após verificar linearidade através de gráficos de dispersão. As taxas de mortalidade nas macrorregiões e entre os sexos foram consideradas variáveis dependentes e os anos variáveis independentes. Incluiu-se o termo quadrático para a variável ano quando foram verificadas tendências curvilíneas em análises gráficas preliminares. Para todas as análises o nível de significância foi fixado em 5\%. A magnitude do crescimento das taxas de mortalidade foram calculadas dividindo-se a taxa no último quinquênio pela taxa no primeiro quinquênio para cada região e para o Brasil.

\section{RESULTADOS}

No gráfico 1, é apresentada a taxa de mortalidade por câncer de fígado e vias biliares entre homens e mulheres. A taxa de mortalidade foi maior entre homens em todos os quinquênios estudados. Observou-se uma tendência de aumento da taxa de mortalidade por esse tipo de câncer ao longo de toda a série histórica em ambos os sexos, com destaque para um maior crescimento no último quinquênio (2010-2014). O crescimento linear entre homens foi de $\beta_{1}=0,67$ (IC95\% 0,26$1,07, \mathrm{p}=0,014)$ e entre mulheres foi $\beta_{1}=0,31$ (IC95\% 0,09-0,53, $\mathrm{p}=0,021$ ).

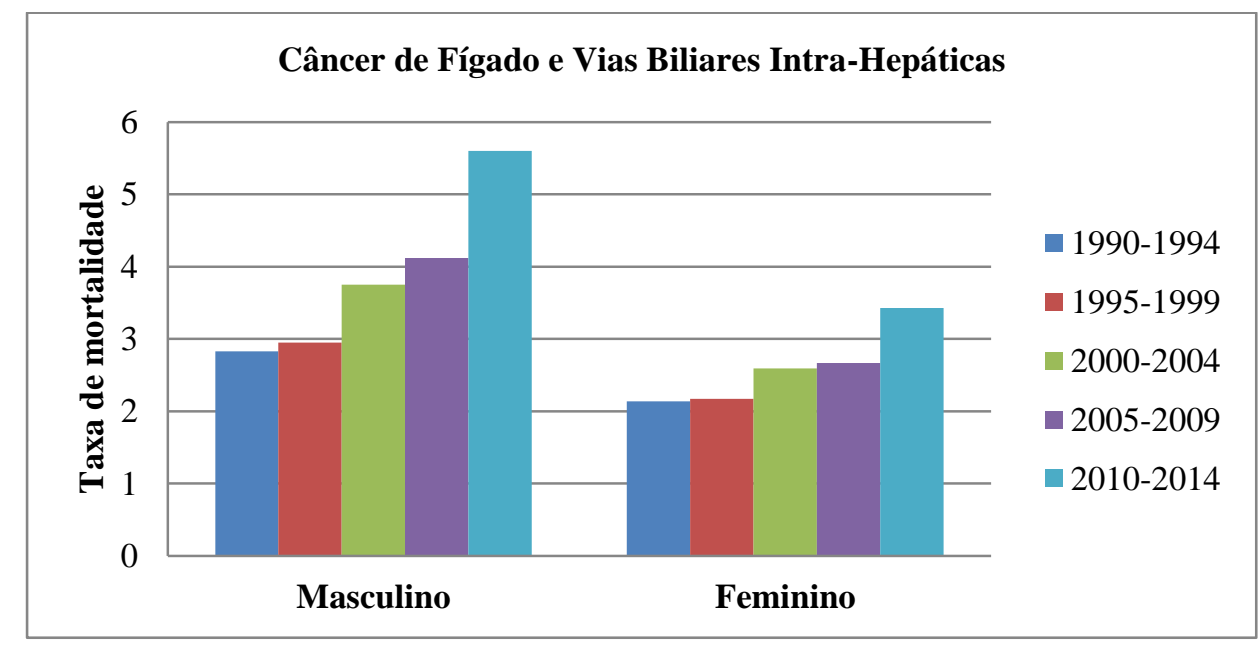

Gráfico 1. Taxa de mortalidade por câncer de fígado e vias biliares intra-hepáticas, ajustada para idade, de acordo com sexo, Brasil, 1990-2014. 
A taxa de mortalidade por câncer colorretal também apresentou crescimento em ambos os sexos ao longo da série histórica, sendo maior entre homens (gráfico 2). A mortalidade por câncer colorretal apresentou tendência de crescimento de $\beta_{1}=0,81(0,60-1,01, p=0,001)$ entre homens e $\beta_{1}=0,58(0,38-0,79, \mathrm{p}=0,003)$ entre mulheres.

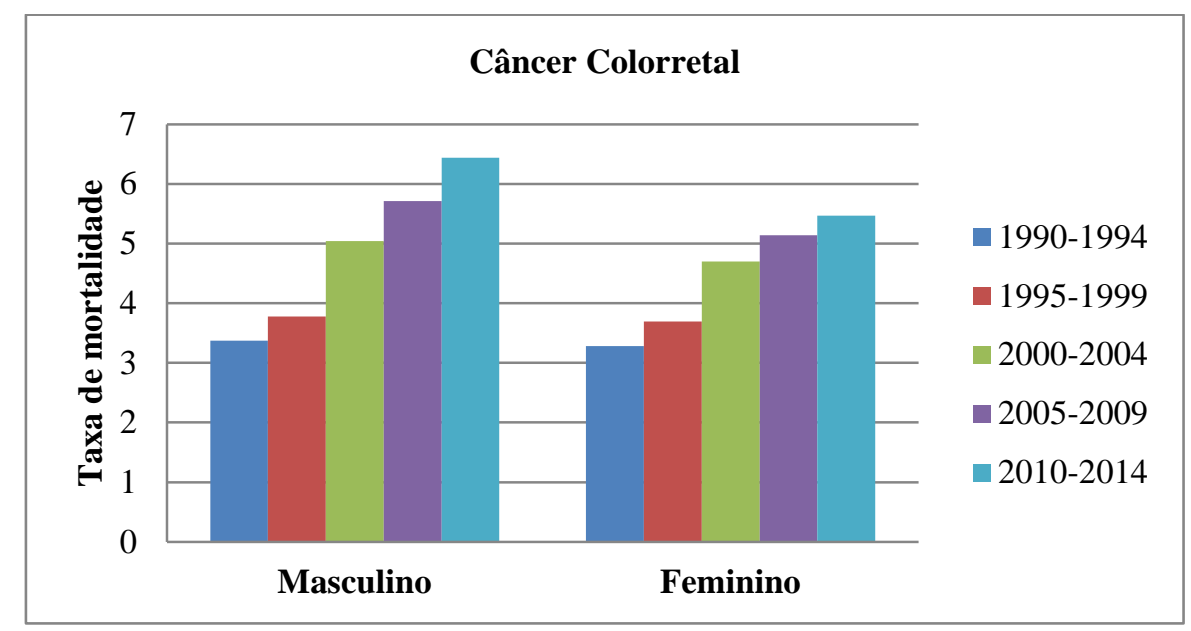

Gráfico 2. Taxa de mortalidade por câncer colorretal, ajustada para idade, de acordo com sexo, Brasil, $1990-2014$.

A tabela 1 mostra as taxas de mortalidade por câncer de fígado e vias biliares e colorretal no Brasil e macrorregiões de 1990 a 2014. O câncer de fígado e vias biliares intra-hepáticas apresentou tendência de aumento no Brasil $\left(\beta_{1}=0,47\right.$, IC95\%=0,17-0,77, $\left.\mathrm{p}=0,016\right)$ e em todas as regiões, com exceção da região Nordeste que não apresentou aumento estatisticamente significante. Embora ao longo de todo o período estudado a taxa de mortalidade tenha sido maior na região Sul, quando comparados o último quinquênio (2010-2014) com o primeiro (1990-1994), observa-se que a taxa de mortalidade cresceu mais nas regiões Norte e Centro-Oeste (1,78 vezes). A região sudeste foi a que apresentou menor crescimento da taxa de mortalidade no período estudado (1,50 vezes). 


\begin{tabular}{lcccccccc}
\hline FíGADO & $\mathbf{1 9 9 0 -}$ & $\begin{array}{r}\mathbf{1 9 9 5}- \\
\mathbf{1 9 9 9}\end{array}$ & $\begin{array}{c}\mathbf{2 0 0 0 -} \\
\mathbf{2 0 0 4}\end{array}$ & $\begin{array}{c}\mathbf{2 0 0 5 -} \\
\mathbf{2 0 0 9}\end{array}$ & $\begin{array}{c}\mathbf{2 0 1 0} \\
\mathbf{2 0 1 4}\end{array}$ & $\boldsymbol{\beta}_{\mathbf{1}}$ & $\mathbf{I C 9 5 \%}$ & $\begin{array}{c}\text { p- } \\
\text { valor }\end{array}$ \\
\hline Norte & 2,47 & 2,50 & 3,21 & 3,24 & 4,40 & 0,46 & $0,12-0,80$ & 0,023 \\
Nordeste & 2,61 & 1,76 & 2,49 & 3,33 & 4,34 & 0,50 & $-0,15-1.16$ & 0,093 \\
Centro-Oeste & 2,37 & 2,93 & 3,32 & 3,25 & 4,22 & 0,40 & $0,14-0,66$ & 0,016 \\
Sudeste & 2,79 & 2,76 & 3,24 & 3,21 & 4,20 & 0,33 & $0,02-0,64$ & 0,044 \\
Sul & 3,12 & 3,17 & 3,38 & 3,77 & 5,07 & 0,45 & $0,01-0,89$ & 0,048 \\
Brasil & 2,47 & 2,54 & 3,13 & 3,34 & 4,41 & 0,47 & $0,17-0,77$ & 0,016 \\
COLORRETAL & & & & & & & & \\
Norte & 1,39 & 1,36 & 1,77 & 2,28 & 3,54 & $-0,63$ & $-1,39-0,14$ & 0,072 \\
Termo quadrático & & & & & & 0,19 & $0,07-0,32$ & 0,022 \\
Nordeste & 1,11 & 1,27 & 1,80 & 2,54 & 3,93 & 0,69 & $0,27-1,11$ & 0,014 \\
Centro-Oeste & 2,55 & 3,11 & 4,41 & 5,28 & 7,59 & 1,23 & $0,69-1,76$ & 0,005 \\
Sudeste & 4,44 & 5,09 & 6,40 & 6,76 & 9,19 & 1,12 & $0,54-1,70$ & 0,009 \\
Sul & 4,92 & 5,21 & 6,95 & 7,18 & 9,80 & 1,17 & $0,47-1,88$ & 0,013 \\
Brasil & 3,32 & 3,74 & 4,86 & 5,40 & 7,48 & 0,99 & $0,49-1,51$ & 0,008 \\
\hline
\end{tabular}

Tabela 1. Taxa de mortalidade por câncer de fígado e vias biliares e colorretal no Brasil e macrorregióes, ajustadas por idade, 1990 a 2014.

Obs: IC95\% - Intervalo de confiança de $95 \%$

O câncer colorretal apresentou tendência de crescimento no Brasil $(\beta=0,99$, IC95\% $=0,49$ 1,51, p=0,008) e em todas as macrorregiões. Na região Norte observou-se tendência de crescimento a partir do segundo quinquênio $(\beta=0,19, \mathrm{IC} 95 \%=0,07-0,32, \mathrm{p}=0,022)$. A região Sul foi a região com maior taxa de mortalidade ao longo de toda a série histórica. Entretanto, na região Nordeste a taxa de mortalidade cresceu 3,5 vezes comparando-se o primeiro com o último quinquênio, contra um crescimento de 1,99 vezes na região Sul.

\section{DISCUSSÕES}

No presente estudo, pôde-se constatar que as taxas de mortalidade por câncer de fígado e colorretal apresentaram tendências de aumento no Brasil ao longo do período estudado, com crescimento maior no último quinquênio (1990-2014). A região Sul foi a que apresentou as maiores taxas de mortalidade para esses tipos de câncer em todos os quinquênios estudados, embora as taxas tenham crescido mais rapidamente em outras regiões. Para ambos os tipos de câncer, a taxa de mortalidade foi maior no sexo masculino ao longo de toda a série histórica.

O câncer de fígado e vias biliares intra-hepáticas é classicamente mais prevalente entre homens, principalmente, porque seus principais fatores de risco ocorrerem com maior frequência nesse sexo (Ferlay et al., 2015; Torre et al., 2016). O consumo excessivo de álcool, apontado como um dos principais fatores de risco para esse tipo de câncer é consideravelmente maior entre homens e apresenta tendência de aumento em todas as regiões do país, com exceção da Sul onde se observou estabilidade (Laranjeira et al.; Schmidt et al., 2011). 
Há que se considerar o fato de que existe desigualdade de gênero na busca por serviços de saúde. É bem documentado que homens tendem a buscar menos os serviços de saúde, fazendo-o apenas em estágios de maior gravidade da doença. Portanto, indivíduos do sexo masculino poderiam estar mais vulneráveis a maior mortalidade, devido ao diagnóstico mais tardio (Laurenti et al., 2005; Moura et al., 2016).

A infecção pelos vírus da hepatite B e C é apontada uma importante causa dos casos de câncer de fígado. Entretanto, no Brasil, a transmissão das hepatites B e C apresenta-se em queda e o país é classificado como de baixa endemicidade (Barreto et al., 2011). Portanto, acredita-se que este fator não esteja contribuindo para o aumento da taxa de mortalidade por câncer de fígado no país. Embora seja importante destacar que a região Norte apresenta soroprevalência do vírus da hepatite C acima da média nacional, o que pode ter contribuído para o maior crescimento das taxas de mortalidade por câncer de fígado nessa região (Guimarães et al., 2015).

A taxa de mortalidade por câncer de fígado e colorretal foi maior na região Sul. Esse resultado é consistente com outros estudos que avaliaram a tendência da mortalidade por esses tipos de câncer no Brasil em outros períodos (GuimarãEs et al., 2012; Amorim e Merchan-Hamann, 2013). A ocorrência de maiores taxas de mortalidade em regiões com melhores condições socioeconômicas seria atribuída ao maior nível de desenvolvimento e a presença de redes de atenção à saúde mais estruturadas, que repercutiriam no maior no rastreamento ou notificação desses tipos de câncer (Amorim e Merchan-Hamann, 2013; Vineis e Wild, 2014).

Por outro lado, uma importante constatação pode ser feita a partir dos resultados do presente estudo. Embora as taxas de mortalidade sejam maiores na região Sul, a magnitude do crescimento ao longo dos anos estudados foi maior na região Norte e Centro-Oeste para câncer de fígado e, no Nordeste, para o câncer colorretal. Esse resultado pode refletir a significativa melhoria ocorrida na qualidade das notificações sobre mortalidade, diagnóstico e redução das causas de óbito mal-definidas (Frias et al., 2010; Amorim e Merchan-Hamann, 2013).

É importante que se considere também as evidências de que os grupos de níveis socioeconômicos mais baixos tendem a apresentar maior prevalência de diagnóstico tardio para neoplasias passíveis de detecção precoce, maior dificuldade de acesso a tratamento adequado, etc (Ribeiro e Nardocci, 2013). Todos esses aspectos implicam em pior prognóstico e menor sobrevida após o diagnóstico, aumentando o risco de mortalidade nas regiões mais pobres do país. Esta tendência de transição na mortalidade por câncer já vem sendo observada quando se compara países de alta renda com países de média e baixa renda, isto é, há maior mortalidade nos países mais pobres e até mesmo redução nos países mais ricos e desenvolvidos (Vineis e Wild, 2014). Portanto, 
é possível que, em alguns anos, se possa observar claramente esse comportamento nas taxas de mortalidade entre as regiões do Brasil.

A dieta parece ter um importante papel no desenvolvimento dos diversos tipos de câncer, incluindo os de fígado e colorretal. Alimentos e nutrientes como vitamina $\mathrm{D}$, cálcio, fibras, leite, frutas, vegetais e grãos integrais parecem reduzir o risco de câncer colorretal (Song et al., 2015). O consumo de frutas, verduras e fibras também está associado ao menor risco de câncer de fígado (Bradbury et al., 2014). Inquéritos nacionais como a Pesquisa de Orçamento Familiares (20082009) e a Pesquisa Nacional de Saúde (2013) apontam dados preocupantes sobre a alimentação da população da brasileira, tais como o baixo consumo de frutas, legumes e verduras, fibras, grãos integrais, bem como elevadas prevalência de inadequação de nutrientes como cálcio, vitaminas A, D, E, entre outros (Ibge, 2010; Claro et al., 2015). Essas constatações a respeito do consumo alimentar da população brasileira apontam para um importante fator de risco que pode estar contribuindo para o aumento das taxas de mortalidade observadas no presente estudo.

É válido destacar ainda o excesso de peso e a inatividade física como importantes fatores de risco para o desenvolvimento desses tipos de câncer (Schmidt et al., 2011). No Brasil, o excesso de peso apresenta tendência ascendente desde a década de 70 , chegando 50,1\% na população adulta masculina de acordo com dados da última Pesquisa de Orçamento Familiares (Ibge, 2010). Quanto a atividade física, apesar de evidências que sugerem um incremento em sua prática, este ainda não é suficiente para compensar o declínio no gasto energético (Schmidt et al., 2011).

O aumento do câncer de fígado e colorretal no Brasil no último quinquênio (2010-2014) foi maior que nos outros períodos analisados. Esse resultado serve de alerta para a necessidade de se implementar estratégias voltadas ao diagnóstico precoce, combate e prevenção desses tipos de câncer no país.

Sabe-se que existem limitações quanto ao uso de sistemas de informação no Brasil, pois apesar das sensíveis melhorias logradas nos últimos anos no aumento da qualidade das informações, ainda existem diferenças na cobertura e completitude dos dados por região. Por isso, é importante que se leve em consideração que a ocorrência de falhas na notificação e definição da causa de morte ainda podem interferir em uma aproximação maior da realidade nacional a partir desses dados. Entretanto, destaca-se que o uso dos sistemas de informação tem sido muito útil para o monitoramento de desfechos em saúde, elaboração e implementação de políticas no país. 


\section{CONCLUSÕES}

Este estudo permitiu conhecer as tendência mais recentes das taxas de mortalidade por câncer de fígado e vias biliares intra-hepáticas e colorretal, em uma série histórica de 25 anos. Foi possível constatar o aumento da velocidade de crescimento nas taxas de mortalidade no quinquênio mais recente (2010 a 2014), período ainda não analisado anteriormente no Brasil. Portanto, os resultados desse estudo são de grande importância para o planejamento de ações a nível nacional, no intuito de combater e prevenir a ocorrência desses tipos de câncer, a fim de conseguir reverter as tendências de crescimento das taxas de mortalidade verificadas no presente trabalho.

\section{REFERÊNCIAS}

AMORIM, T. R.; MERCHAN-HAMANN, E. [Mortality due to malignant neoplasms of the liver and intrahepatic bile ducts in Brazil, 1980-2010]. Cad Saude Publica, v. 29, n. 7, p. 1427-36, Jul 2013. Disponível em: < https://www.ncbi.nlm.nih.gov/pubmed/23843009>.

BARRETO, M. L. et al. Successes and failures in the control of infectious diseases in Brazil: social and environmental context, policies, interventions, and research needs. Lancet, v. 377, n. 9780, p. 1877-89, 2011. Disponível em: < https://www.ncbi.nlm.nih.gov/pubmed/21561657 >.

BRADBURY, K. E.; APPLEBY, P. N.; KEY, T. J. Fruit, vegetable, and fiber intake in relation to cancer risk: findings from the European Prospective Investigation into Cancer and Nutrition (EPIC). Am J Clin Nutr, v. 100 Suppl 1, p. 394S-8S, Jul 2014. Disponível em: < https://www.ncbi.nlm.nih.gov/pubmed/24920034 >.

CLARO, R. M. et al. Consumo de alimentos não saudáveis relacionados a doenças crônicas não transmissíveis no Brasil: Pesquisa Nacional de Saúde, 2013. Epidemiologia e Serviços de Saúde, v. 24, p. 257-265, 2015. ISSN 2237-9622. Disponível em: < http://www.scielo.br/scielo.php?script=sci_arttext\&pid=S2237-96222015000200257\&nrm=iso >.

FERLAY, J. et al. Cancer incidence and mortality worldwide: sources, methods and major patterns in GLOBOCAN 2012. Int J Cancer, v. 136, n. 5, p. 359-86, 2015. Disponível em: < https://www.ncbi.nlm.nih.gov/pubmed/25220842 >.

FRIAS, P. G. et al. [Evaluation of data on mortality and live births in Pernambuco State, Brazil]. Cad Saude Publica, v. 26, n. 4, p. 671-81, Apr 2010. Disponível em: < https://www.ncbi.nlm.nih.gov/pubmed/20512208 >.

GIRIANELLI, V. R.; GAMARRA, C. J.; AZEVEDO E SILVA, G. Disparities in cervical and breast cancer mortality in Brazil. Rev Saude Publica, v. 48, n. 3, p. 459-67, 2014. Disponível em: < https://www.ncbi.nlm.nih.gov/pubmed/25119941 >.

GUIMARÃES, R. M. et al. Tendência para o câncer de fígado e vias biliares na Região Norte do Brasil. Revista Pan-Amazônica de Saúde, v. 6, p. 29-34, 2015. Disponível em: < http://scielo.iec.pa.gov.br/scielo.php?script=sci_arttext\&pid=S2176-62232015000100004\&nrm=iso $>$. 
GUIMARÃES, R. M. A. et al. Tendência da mortalidade por câncer de cólon e reto no Brasil segundo sexo, 1980-2009. Cadernos Saúde Coletiva, v. 20, n. 1, p. 7, 2012.

IBGE. Instituto Brasileiro de Geografia e Estatística. Pesquisa de orçamentos familiares, 20082009. Análise do consumo alimentar pessoal no Brasil. Rio de Janeiro. . 2010.

LARANJEIRA, R. et al. II Levantamento Nacional de Álcool e Drogas - Consumo de Álcool no Brasil: Tendências entre 2006/2012. São Paulo: INPAD; 2013. Disponível em:< http://inpad.org.br/wp-content/uploads/2013/04/LENAD_ALCOOL_Resultados-Preliminares.pdf > Acesso em 31 março 2017. .

LAURENTI, R.; JORGE, M. H. P. D. M.; GOTLIEB, S. L. D. Perfil epidemiológico da morbimortalidade masculina. Ciência \& Saúde Coletiva, v. 10, p. 35-46, 2005. Disponível em: < http://www.scielo.br/scielo.php?script=sci_arttext\&pid=S1413-81232005000100010\&nrm=iso >.

MOURA, E. C. D. et al. Mortality in Brazil according to gender perspective, years 2000 and 2010. Revista Brasileira de Epidemiologia, v. 19, p. 326-338, 2016. Disponível em: < http://www.scielosp.org/scielo.php?script=sci_arttext\&pid=S1415-790X2016000200326\&nrm=iso $>$.

RIBEIRO, A. D. A.; NARDOCCI, A. C. Desigualdades socioeconômicas na incidência e mortalidade por câncer: revisão de estudos ecológicos, 1998-2008. Saúde e Sociedade, v. 22, p. 878-891, 2013. Disponível em: < http://www.scielo.br/scielo.php?script=sci_arttext\&pid=S0104$12902013000300020 \&$ nrm $=$ iso $>$.

SCHMIDT, M. I. et al. Chronic non-communicable diseases in Brazil: burden and current challenges. Lancet, v. 377, n. 9781, p. 1949-61, 2011. Disponível em: < https://www.ncbi.nlm.nih.gov/pubmed/21561658 >.

SONG, M.; GARRETT, W. S.; CHAN, A. T. Nutrients, foods, and colorectal cancer prevention. Gastroenterology, v. 148, n. 6, p. 1244-60 May 2015. Disponível em: < https://www.ncbi.nlm.nih.gov/pubmed/25575572 >.

TORRE, L. A. et al. Global Cancer Incidence and Mortality Rates and Trends--An Update. Cancer Epidemiol Biomarkers Prev, v. 25, n. 1, p. 16-27, Jan 2016. Disponível em: < https://www.ncbi.nlm.nih.gov/pubmed/26667886>.

VINEIS, P.; WILD, C. P. Global cancer patterns: causes and prevention. Lancet, v. 383, n. 9916, p. 549-57, 2014. Disponível em: < https://www.ncbi.nlm.nih.gov/pubmed/24351322 >. 